\title{
Probing cosmic chemical evolution with gamma-ray bursts: GRB 060206 at $z=4.048^{\star}$
}

\author{
J. P. U. Fynbo ${ }^{1}$, R. L. C. Starling ${ }^{2}$, C. Ledoux ${ }^{3}$, K. Wiersema ${ }^{2}$, C. C. Thöne ${ }^{1}$, J. Sollerman ${ }^{1}$, P. Jakobsson ${ }^{1}$, J. Hjorth ${ }^{1}$, \\ D. Watson ${ }^{1}$, P. M. Vreeswijk ${ }^{4}, 3$, P. Møller ${ }^{5}$, E. Rol ${ }^{6}$, J. Gorosabel ${ }^{7}$, J. Näränen ${ }^{8}$, R. A. M. J. Wijers ${ }^{2}$, G. Björnsson ${ }^{9}$, \\ J. M. Castro Cerón ${ }^{1}$, P. Curran ${ }^{2}$, D. H. Hartmann ${ }^{10}$, S. T. Holland ${ }^{11}$, B. L. Jensen ${ }^{1}$, A. J. Levan ${ }^{12}$, M. Limousin ${ }^{1}$, \\ C. Kouveliotou ${ }^{13}$, G. Nelemans ${ }^{14}$, K. Pedersen ${ }^{1}$, R. S. Priddey ${ }^{12}$, and N. R. Tanvir ${ }^{12}$ \\ (Affiliations can be found after the references)
}

Received 20 February 2006 / Accepted 2 April 2006

\begin{abstract}
Aims. We present early optical spectroscopy of the afterglow of the gamma-ray burst GRB 060206 with the aim of determining the metallicity of the GRB absorber and the physical conditions in the circumburst medium. We also discuss how GRBs may be important complementary probes of cosmic chemical evolution.

Methods. Absorption line study of the GRB afterglow spectrum.

Results. We determine the redshift of the GRB to be $z=4.04795 \pm 0.00020$. Based on the measurement of the neutral hydrogen column density from the damped Lyman- $\alpha$ line and the metal content from weak, unsaturated $\mathrm{S}$ II lines we derive a metallicity of $[\mathrm{S} / \mathrm{H}]=-0.84 \pm 0.10$. This is one of the highest metallicities measured from absorption lines at $z \sim 4$. From the very high column densities for the forbidden Si II*, $\mathrm{OI}^{*}$, and $\mathrm{OI}^{* *}$ lines we infer very high densities and low temperatures in the system. There is evidence for the presence of $\mathrm{H}_{2}$ molecules with $\log N\left(\mathrm{H}_{2}\right) \sim 17.0$, translating into a molecular fraction of $\log f \approx-3.5$ with $f=2 N\left(\mathrm{H}_{2}\right) /\left(2 N\left(\mathrm{H}_{2}\right)+N(\mathrm{H} \mathrm{I})\right)$. Even if GRBs are only formed by single massive stars with metallicities below $\sim 0.3 Z_{\odot}$, they could still be fairly unbiased tracers of the bulk of the star formation at $z>2$. Hence, metallicities as derived for GRB 060206 here for a complete sample of GRB afterglows will directly show the distribution of metallicities for representative star-forming galaxies at these redshifts.
\end{abstract}

Key words. gamma rays: bursts - galaxies: high-redshift - galaxies: abundances - cosmology: observations

\section{Introduction}

Long gamma-ray bursts (GRBs) are now established to be caused by the deaths of massive stars (e.g., Hjorth et al. 2003; Stanek et al. 2003) and due to their brightness they can be observed throughout most of the observable Universe (e.g., Kawai et al. 2006). Given these facts it has long been realized that GRBs could be ideal probes of star-formation activity throughout the history of the Universe (e.g., Wijers et al. 1998). However, previous GRB missions detected too few rapidly welllocalised GRBs to build statistically interesting samples and hence really capitalize on this potential. The Swift satellite (Gehrels et al. 2004) has increased the detection rate of rapidly well-localised GRBs by roughly an order of magnitude compared to previous missions. Moreover, its significantly deeper detection limit (e.g., Band 2006) means that Swift detects more distant bursts than previous missions (Jakobsson et al. 2006).

In this Letter we present optical spectroscopy of GRB 060206, focussing on the measurement of the metallicity of the GRB absorption system, the density in the circumburst environment and the presence of molecules. We then discuss how GRBs are important for understanding the build-up and distribution of metals in galaxies (e.g., Pei \& Fall 1995), which is currently not fully understood (e.g., Ferrara et al. 2005).

^ Based on observations made at the Spanish Observatorio del Roque de los Muchachos on La Palma with the Nordic Optical Telescope, operated jointly by Denmark, Finland, Iceland, Norway, and Sweden, and with the William Herschel Telescope.

\section{Observations}

GRB 060206 was discovered by the Burst Alert Telescope (BAT) aboard the Swift satellite on February 6 04:46:53 UT. The burst exhibited a slow rise and a faster decline, with a $T_{90}$ of $7 \pm 2$ s (Palmer et al. 2006). The X-ray Telescope (XRT) slewed promptly to the location and began taking data at $\Delta t=58 \mathrm{~s}$, where $\Delta t$ is the time from the onset of the burst. Due to entry into the South Atlantic Anomaly, XRT could only observe the BAT error circle briefly, and therefore no fading X-ray source was immediately localised (Morris et al. 2006a). Observations with the UV/Optical Telescope (UVOT) began at $\Delta t=57 \mathrm{~s}$ but failed to reveal an optical afterglow (OA) candidate in the initial data products (Morris et al. 2006a).

We observed the GRB 060206 BAT error circle in the $R$ band with the Andalucía Faint Object Spectrograph and Camera (ALFOSC) on the Nordic Optical Telescope (NOT) starting at $\Delta t \approx 15 \mathrm{~min}$. A point-like object $(R \sim 17.3)$ not present in the Digitized Sky Survey was detected. The detection was confirmed by re-analysis of the XRT and UVOT data (Boyd et al. 2006; Morris et al. 2006b).

Starting at $\Delta t \approx 48 \mathrm{~min}$ we obtained a $1800 \mathrm{~s}$ spectrum with a low resolution (LR) grism and a 1.3 arcsec wide slit covering the spectral range from about $3500 \AA$ to $9000 \AA$ at a resolution of $14 \AA$. The airmass during the spectroscopic observation was very low, starting at 1.01 . We also obtained medium resolution (MR) spectroscopic data with the Intermediatedispersion Spectroscopic and Imaging System (ISIS) on the William Herschel Telescope (WHT) at $\Delta t=1.61 \mathrm{~h}$. We took 
spectra of exposure times $2 \times 900 \mathrm{~s}$ with both the the $600 \mathrm{~B}$ grating (covering 3800-5300 $\AA$ ) and and the $1200 \mathrm{R}$ grating (covering 6100-7200 $\AA$ ) both with a 1.0 arcsec wide slit. The mean airmass was 1.036 and the observations were carried out at the parallactic angle. The resolutions of blue- and red-arm spectra are 1.68 and $0.82 \AA$, respectively.

\section{Results}

The spectra show absorption both at the GRB redshift of $z=$ $4.04795 \pm 0.00020$ and from two intervening systems at redshifts $z=1.48$ and $z=2.26$. In the analysis presented here we focus on the metallicity of the GRB absorption system. From a fit of the damped Ly $\alpha$ line at $z=4.048$, we measure a neutral hydrogen column density of $\log N(\mathrm{HI})=20.85 \pm 0.10$ (Fig. 1), well above the definition for Damped Ly $\alpha$ Absorbers (DLAs, $\log N(\mathrm{HI}) \geq$ 20.3). This value is consistent with fits to the higher order Lyman lines in the blue MR spectrum. To derive the metal column density we use the unsaturated S II $\lambda 1250,1253,1259$ lines shown in Fig. 1. The profile of the GRB absorption systems consists of at least four components spread over $\sim 500 \mathrm{~km} \mathrm{~s}^{-1}$ in velocity space. On the right hand side of the middle panel of Fig. 1, a four-component fit of S II and Si II* is shown. On the left hand side, we have only fitted the redmost component at $175 \mathrm{~km} \mathrm{~s}^{-1}$ where we can derive a column density for Si II, O I* and O I**. We fit the lines using the FitLyman package in MIDAS; the measured column densities for all components are given in Table 1. Note that for components 2 and 3 we fixed the turbulent broadening parameter value to $15 \mathrm{~km} \mathrm{~s}^{-1}$. Summing over the four components of the system, we get $\log N(\mathrm{~S}$ II $)=15.21 \pm 0.03$ which leads to $[\mathrm{S} / \mathrm{H}]=-0.84 \pm 0.10$.

We also find $\log N\left(\mathrm{Si}_{\mathrm{II}}^{*}\right)=14.42 \pm 0.02$ and therefore $\left[\mathrm{Si} \mathrm{II}^{*} / \mathrm{Si} \mathrm{II}\right]=-1.15$, assuming $[\mathrm{S} / \mathrm{Si}]=0$. In the fourth component, we have $\left[\mathrm{Si} \mathrm{II}^{*} / \mathrm{Si} \mathrm{II}\right]=-0.34$, which is high compared to the ratios found in other GRB host galaxies (twice as high as the value found for GRB 050505 by Berger et al. 2005 and 20 times higher than the value found for GRB 030323 by Vreeswijk et al. 2004). Fine-structure levels can be populated through collisions, photo-excitation by IR photons, and/or fluorescence (Bahcall \& Wolf 1968). Assuming that the former mechanism is dominant (but see Berger et al. 2006; Prochaska et al. 2006), we can estimate the H I volume density, using the calculations by Silva \& Viegas (2002). Assuming an electron fraction $n_{\mathrm{e}} \sim 10^{-4} n_{\mathrm{HI}}$ (see Vreeswijk et al. 2004; Berger et al. 2005) and a temperature of $1000 \mathrm{~K}$, we find $n_{\mathrm{HI}} \sim 10^{5} \mathrm{~cm}^{-3}$ for the fourth component, and $n_{\mathrm{HI}} \sim 10^{4} \mathrm{~cm}^{-3}$ for the mean ratio of all components (see Fig. 8 of Silva \& Viegas 2002). For the fourth component, we can actually constrain the kinetic temperature and volume density, as the observed ratio $\left[\mathrm{OI}^{*} / \mathrm{OI}^{* *}\right]=0.78 \pm 0.17$ can only be reached below $320 \mathrm{~K}$ (within the $1 \sigma$ errors), while $\left[\mathrm{Si} \mathrm{II}^{*} / \mathrm{Si} \mathrm{II}\right]=-0.34 \pm 0.11$ requires a temperature above $240 \mathrm{~K}$. This temperature range corresponds to a volume density of $n_{\mathrm{HI}}=$ $1-3 \times 10^{7} \mathrm{~cm}^{-3}$.

Finally, our data show the first evidence for $\mathrm{H}_{2}$ molecules in a GRB absorber. Two consistent features are seen at the location of the W1-0 R(0), W1-0 R(1) and W1-0 Q(1) lines at $z_{\mathrm{abs}}=4.04793$ (bottom panel in Fig. 1), with column densities of $\log N\left(\mathrm{H}_{2}\right) \sim 17.0$ for the $J=1$ rotational level, and $\log N\left(\mathrm{H}_{2}\right)<16.7$ for $J=0$. The corresponding $\mathrm{H}_{2}$ molecular fraction is $\log f \sim-3.5$ with $f=2 N\left(\mathrm{H}_{2}\right) /\left(2 N\left(\mathrm{H}_{2}\right)+N(\mathrm{HI})\right)$. This is the second highest redshift at which these $\mathrm{H}_{2}$ lines have been detected (Ledoux et al. 2006).
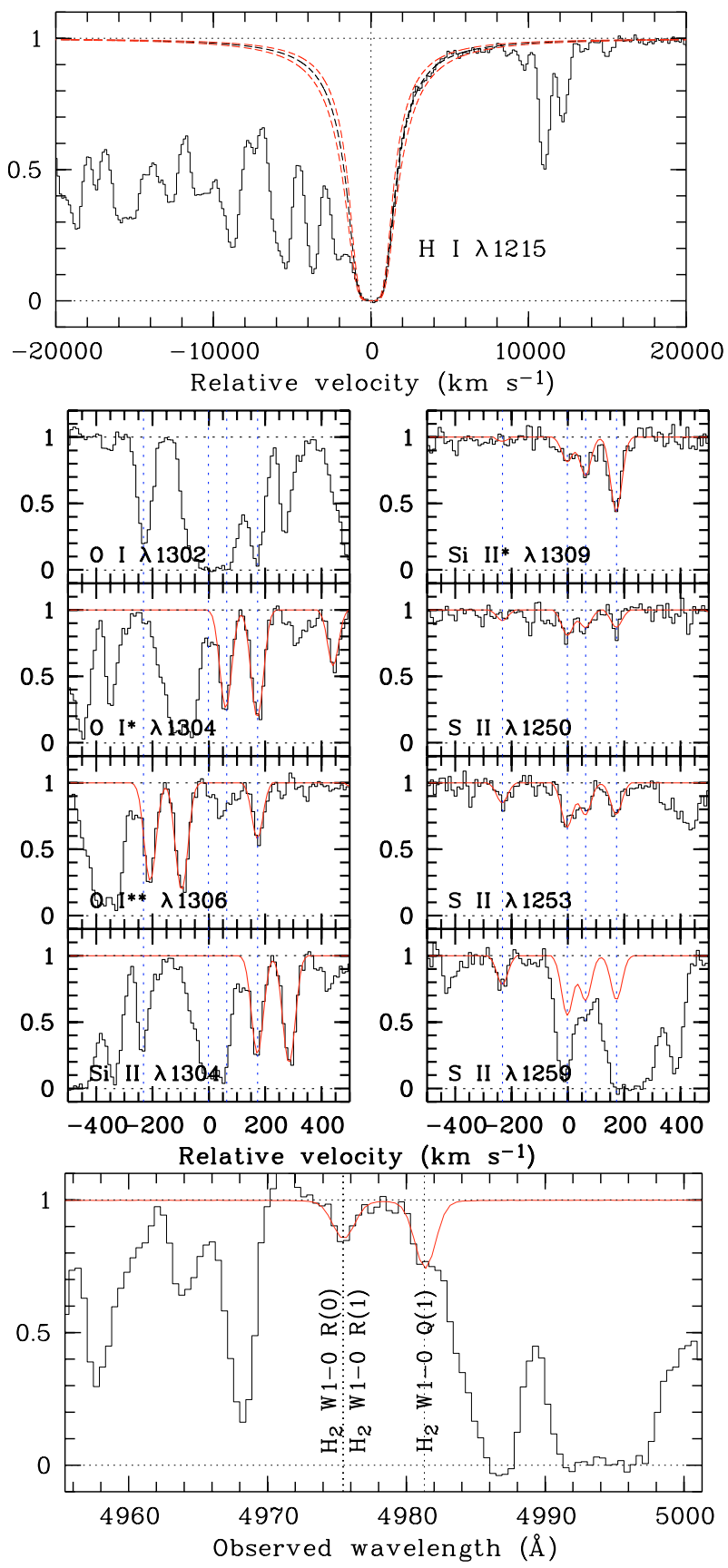

Fig. 1. Upper panel: section of the LR afterglow spectrum showing the DLA line at the GRB redshift, $z_{\mathrm{abs}}=4.048$. Overlaid is the best fitting DLA profile, corresponding to $\log N(\mathrm{H} \mathrm{I})=20.85 \pm 0.10$. Middle panel: fits to the O I, O I*, O I**, Si II, Si II*, and S II lines in the MR spectrum. The zero-point for the velocity scale is $z_{\text {abs }}=4.048$. Lower panel: the likely $\mathrm{H}_{2}$ lines at the GRB redshift in the MR spectrum.

\section{Discussion and conclusions}

In Table 2, we have compiled metallicity measurements for $z>$ 2 GRB absorption systems from the literature. As seen, despite its high redshift, the GRB 060206 system has one of the highest metallicities measured for a GRB absorption system and one of the highest metallicities measured from QSO absorption lines at $z>4$. We also note that the metallicity of GRB 060206 is about 15 times higher than that of GRB 050730 which has a similar redshift $(z=3.968)$. This shows that GRBs do not only occur 
Table 1. Ionic column densities in individual components of the GRB system at $z_{\mathrm{abs}}=4.048$.

\begin{tabular}{|c|c|c|c|}
\hline Ion & $\begin{array}{l}\text { Transition } \\
\text { lines used }\end{array}$ & $\overline{\log N \pm \sigma_{\log N}}$ & $\begin{array}{l}b \pm \sigma_{b} \\
\left(\mathrm{~km} \mathrm{~s}^{-1}\right)\end{array}$ \\
\hline \multicolumn{4}{|c|}{$z_{\mathrm{abs}}=4.0441$} \\
\hline S II & $1250,1253,1259$ & $14.36 \pm 0.06$ & $14 \pm 7$ \\
\hline Si II* & 1309 & $<13.10^{a}$ & $14 \pm 7$ \\
\hline \multicolumn{4}{|c|}{$z_{\mathrm{abs}}=4.0480$} \\
\hline S II & $1250,1253,1259$ & $14.79 \pm 0.05$ & $\sim 15$ \\
\hline Si II* & 1309 & $13.54 \pm 0.08$ & $\sim 15$ \\
\hline \multicolumn{4}{|c|}{$z_{\mathrm{abs}}=4.0490$} \\
\hline S II & $1250,1253,1259$ & $14.60 \pm 0.06$ & $\sim 15$ \\
\hline Si II* & 1309 & $13.77 \pm 0.06$ & $\sim 15$ \\
\hline \multicolumn{4}{|c|}{$z_{\mathrm{abs}}=4.0509$} \\
\hline S II & $1250,1253,1259$ & $14.59 \pm 0.06$ & $14 \pm 2$ \\
\hline Si II* & 1309 & $14.22 \pm 0.05$ & $14 \pm 2$ \\
\hline OI* & 1304 & $15.03 \pm 0.16$ & $14 \pm 2$ \\
\hline OI** & 1306 & $14.25 \pm 0.05$ & $14 \pm 2$ \\
\hline Si II & 1304 & $14.56 \pm 0.10$ & $14 \pm 2$ \\
\hline
\end{tabular}

a $3 \sigma$ upper limit.

Table 2. Table of published absorption metallicities for GRBs.

\begin{tabular}{lllr}
\hline \hline GRB & Metallicity & Redshift & Ref. \\
\hline 000926 & {$[\mathrm{Zn} / \mathrm{H}]=-0.13 \pm 0.25$} & 2.038 & 1 \\
011211 & {$[\mathrm{Si} / \mathrm{H}]=-0.90 \pm 0.5$} & 2.142 & 2 \\
030323 & {$[\mathrm{~S} / \mathrm{H}]=-1.26 \pm 0.2$} & 3.372 & 3 \\
050401 & {$[\mathrm{Zn} / \mathrm{H}]=-1.0 \pm 0.4$} & 2.899 & 4 \\
050505 & {$[\mathrm{~S} / \mathrm{H}] \gtrsim-1.2$} & 4.275 & 5 \\
050730 & {$[\mathrm{~S} / \mathrm{H}]=-2.0 \pm 0.2$} & 3.968 & 6,7 \\
050820 & {$[\mathrm{Si} / \mathrm{H}]=-0.6 \pm 0.1$} & 2.615 & 8 \\
050904 & {$[\mathrm{~S} / \mathrm{H}]=-1.3 \pm 0.3$} & 6.295 & 9 \\
060206 & {$[\mathrm{~S} / \mathrm{H}]=-0.84 \pm 0.10$} & 4.048 & 10 \\
\hline
\end{tabular}

References: [1] Savaglio et al. (2003); [2] Vreeswijk et al. (2006); [3] Vreeswijk et al. (2004); [4] Watson et al. (2006); [5] Berger et al. (2005); [6] Starling et al. (2005); [7] Chen et al. (2005); [8] Ledoux et al. (2005); [9] Kawai et al. (2005); [10] this work.

in very low metallicity environments, but also in environments covering a broad range of metallicities at a given redshift.

Fine-structure lines are ubiquitous in GRB absorbers (Vreeswijk et al. 2004; Berger et al. 2005; Chen et al. 2005). As discussed above, for GRB 060206 we derive high densities and low temperatures, consistent with the possible detection of $\mathrm{H}_{2}$ in the spectrum, with a molecular fraction of $\log f \sim$ -3.5. Molecules are also detected in QSO-DLAs (Ledoux et al. $2003)$ in cold gas with $T \lesssim 300 \mathrm{~K}$, but with lower densities ( $\$ 400 \mathrm{~cm}^{-3}$, Srianand et al. 2005). The difference in densities along random (cross-section selected) and GRB sightlines like the one towards GRB 060206 must reflect the higher than average density of the star forming region in which the progenitor star was located. Such regions must have a cross-section which is less than a few percent of the total cross-section for QSO-DLAs at redshifts $z=2-4$ in order to explain that similar fine-structure lines have not yet been seen in QSO-DLAs.

In the collapsar models GRBs can only be formed by massive single stars with a metallicity below $\sim 0.3 Z_{\odot}$ (Hirschi et al. 2005; Woosley \& Heger 2006). Such a bias is not present in all models, most noteably not in models involving a binary progenitor (Fryer \& Heger 2005). To gauge what a metallicity bias could mean for the completeness of GRBs as cosmological probes it is important to know the present mass-weighted mean metallicity and how it declines with redshift. Zwaan et al. (2005) find a



Fig. 2. Metallicity as a function of redshift for different classes of objects. The black circles are the measurements for GRBs from Table 2 (GRB 060206 is also marked with $\times$ ). The open triangles show the metallicity of three low- $z$ GRB host galaxies (Sollerman et al. 2005). The squares and the dashed line represent the column density weighted metallicity evolution derived by Zwaan et al. (2005, their Fig. 22). The small dots with no error-bars are measurements for 121 DLAs from Prochaska et al. (2003). The hatched region indicates the metallicity above which GRBs cannot form in the collapsar models.

present-day mean metallicity in the gas phase of $\bar{Z} \approx 0.44 Z_{\odot}$ and a slope between -0.25 and -0.30 dex per unit redshift. This means that at $z \approx 1$ and earlier the mean metallicity of the gas is below the cut-off value above which single massive stars in the collapsar models do not make GRBs. It is therefore likely that GRBs at $z>2$ will be fairly unbiased tracers of star-formation, while they become increasingly biased at $z<1$ if there is a lowmetallicity bias.

Metallicities can be measured from optical spectroscopy of the afterglow or the host galaxy. Figure 2 shows that most metallicities for GRB absorption systems fall below $0.3 Z_{\odot}$, but one (GRB 000926, $Z=0.7 Z_{\odot}$, Savaglio et al. 2003) has a metallicity well above the threshold for a single massive star to produce GRBs in the collapsar models. The metallicity of the host galaxy of GRB 980425 by Sollerman et al. (2005) is also higher than this limit $\left(0.8 Z_{\odot}\right)$. This indicates that collapsars resulting from single massive stars are not the only progenitors to long GRBs or that massive stars with $Z>0.3 Z_{\odot}$ can also produce long GRBs. The GRB metallicities fall within the range spanned by QSO-DLAs, but most are above the mean curve derived by Zwaan et al. (2005). The mean offset is 0.49 and the scatter 0.38 dex (excluding GRB 050904 which is at very high redshift where there are no QSO-DLA data). This offset may reflect that DLAs are cross-section selected, whereas GRBs represent sightlines to more central regions in their hosts (Bloom et al. 2002). A metallicity gradient of $-0.09 \mathrm{dex} \mathrm{kpc}^{-1}$ as assumed in the study by Zwaan et al. (2005) (and observed in the Galaxy) seems sufficient to explain the offset. Note that the current sample of QSO-DLAs is not likely to be strongly biased against high metallicity sightlines (Akerman et al. 2005).

Furthermore, in the derivation of the cosmic star-formation density, and hence the production of metals, the shape of the luminosity function is important. Roughly, two thirds of the UV light from LBGs is emitted by galaxies that are fainter than the actual flux limit of the ground based LBG survey of Steidel et al. (2003). Using only LBGs to derive the total star-formation density means that an extrapolation to the poorly determined 
faint end of the luminosity function is unavoidable. GRBs allow us to probe this faint end since the selection is not limited by the brightness of the host. We refer to Jakobsson et al. (2005) for a quantitative analysis. Finally, GRBs allow the measurement of metallicities at very high redshifts ( $z>6$, Kawai et al. 2006), which is inaccessible to QSO-DLAs.

In conclusion, we measure a metallicity of $[\mathrm{S} / \mathrm{H}]=-0.84 \pm$ 0.10 for GRB 060206 at $z=4.048$. We also find the first evidence for molecular lines from a GRB absorber. We have argued that GRBs can be used to measure the metallicities and luminosities of typical star forming galaxies at $z>2$, making GRBs promising complementary probes of chemical evolution at high redshift.

Acknowledgements. The authors acknowledge the indispensable assistance given by both observers and staff at WHT and NOT. We also thank C. Péroux, S. Ellison, and J. Andersen for helpful discussions. The Dark Cosmology Centre is funded by the Danish Nationa Research Foundation. K.W. and P.C. thank NWO for support under grant 639.043.302. A.L., N.R.T. and E.R. thank PPARC for support. The research of J.G. is supported by the Spanish Ministry of Science and Education through programmes ESP2002-04124-C03-01 and AYA2004-01515. J.M.C.C. acknowledges partial support from IDA and the NBI' International Ph.D. School of Excellence. We also acknowledge benefits from collaboration within the EU FP5 Research Training Network "Gamma-Ray Bursts: An Enigma and a Tool" (HPRN-CT-2002-00294).

\section{References}

Akerman, C. J., Ellison, S. L., Pettini, M., \& Steidel, C. C. 2005, A\&A, 440, 499 Bahcall, J. N., \& Wolf, R. A. 1968, ApJ, 152, 701

Band, D. L. 2006, ApJ, in press [arXiv: astro-ph/0602267]

Berger, E., Penprase, B. E., Cenko, S. B., et al. 2005, ApJ, submitted [arXiv: astro-ph/0511498]

Bloom, J. S., Kulkarni, S. K., \& Djorgovski, S. G. 2002, AJ, 123, 1111

Boyd, P., Morris, D., Marshall, F., \& Gehrels, N. 2006, GCN Circ., 4722

Chen, H.-W., Prochaska, J. X., Bloom, J. S., \& Thompson, I. B. 2005, ApJ, 634, L25

Ferrara, A., Scannapieco, E., \& Bergeron, J. 2005, ApJ, 634, L37

Fryer, C. L., \& Heger, A. 2005, ApJ, 623, 302

Gehrels, N., Chincarini, G., Giommi, P., et al. 2004, ApJ, 611, 1005

Heger, A., Fryer, C. L., Woosley, S. E., Langer, N., \& Hartmann, D. H. 2003, ApJ, 591, 288 Hirschi, R., Meynet, G., \& Maeder, A. 2005, A\&A, 443, 581

Hjorth, J., Sollerman, J., Møller, P., et al. 2003, Nature, 423, 847

Jakobsson, P., Björnsson, G., Fynbo, J. P. U., et al. 2005, MNRAS, 362, 245

Jakobsson, P., Levan, A., Fynbo, J. P. U., et al. 2006, A\&A, 447, 897

Kawai, N., Kosugi, G., Aoki, K., et al. 2005, Nature, 440, 184

Ledoux, C., Petitjean, P., \& Srianand, R. 2003, MNRAS, 346, 209

Ledoux, C., Vreeswijk, P., Ellison, S., et al. 2005, GCN Circ., 3860

Ledoux, C., Petitjean, P., \& Srianand, R. 2006, ApJL, in press [arXiv : astro-ph/0602212]
Morris, D. C., Barbier, L., \& Barthelmy, S. 2006a, GCN Circ., 4682

Morris, D. C., Burrows, D., Gehrels, N., Boyd, P., \& Angelini, L. 2006b, GCN Circ., 4689 Palmer, D., Barbier, L., Barthelmy, S., et al. 2006, GCN Circ., 4697

Pei, Y. C., \& Fall, M. S. 1995, ApJ, 454, 69

Prochaska, J. X., Gawiser, E., Wolfe, A. M., Castro, S., \& Djorgovski, S. G. 2003, ApJ, 595, L9

Prochaska, J. X., Chen, H.-W., \& Bloom, J. S. 2006, ApJ, submitted [arXiv: astro-ph/0601057]

Silva, A. I., \& Viegas, S. M. 2002, MNRAS, 329, 135

Sollerman, J., Östlin, G., Fynbo, J. P. U., et al. 2005, NewA, 11, 103

Srianand, R., Petitjean, P., Ledoux, C., Ferland, G., \& Shaw, G. 2005, MNRAS, 362, 549

Stanek, K. Z., Matheson, T., Garnavich, P. M., et al. 2003, ApJ, 591, L17

Starling, R. L. C., Vreeswijk, P. M., Ellison, S. L., et al. 2005, A\&A, 442, L21

Savaglio, S., Fall, S. M., \& Fiore, F. 2003, ApJ, 585, 638

Vreeswijk, P. M., Ellison, S. L., Ledoux, C., et al. 2004, A\&A, 419, 927

Vreeswijk, P. M., Smette, A., Fruchter, A. S., et al. 2006, A\&A, 447, 145

Wijers, R. A. M. J., Bloom, J. S., Bagla, J. S., \& Natarajan, P. 1998, MNRAS, 297, L13

Woosley, S. E., \& Heger, A. 2006, ApJ, 637, 914

Zwaan, M. A., van der Hulst, J. M., Briggs, F. H., Verheijen, M. A. W., \& Ryan-Weber, E. V. 2005, MNRAS, 364, 1467

1 Dark Cosmology Centre, Niels Bohr Institute, University of Copenhagen, Juliane Maries Vej 30, 2100 Copenhagen, Denmark e-mail: jfynbo@astro.ku.dk

2 Astronomical Institute 'Anton Pannekoek', University of Amsterdam, Kruislaan 403, 1098 SJ Amsterdam, The Netherlands

3 European Southern Observatory, Alonso de Córdova 3107, Casilla 19001, Vitacura, Santiago, Chile

${ }^{4}$ Departamento de Astronomía, Universidad de Chile, Casilla 36-D, Santiago, Chile

5 European Southern Observatory, Karl-Schwarzschild-strasse 2, 85748 Garching bei München, Germany

6 Department of Physics and Astronomy, University of Leicester, University Road, Leicester LE1 7RH, UK

7 Instituto de Astrofísica de Andalucía (CSIC), Apartado de Correos 3004, 18080 Granada, Spain

8 Observatory, University of Helsinki, PO Box 14, 00014 Helsinki, Finland

9 Science Institute, University of Iceland, Dunhaga 3, 107 Reykjavík, Iceland

10 Department of Physics and Astronomy, Clemson University, Clemson, South Carolina 29634-0978, USA

11 NASA Goddard Space Flight Center, Greenbelt, MD 20771, USA

12 Centre for Astrophysics Research, University of Hertfordshire, College Lane, Hatfield, Hertfordshire AL10 9AB, UK

13 NASA Marshall Space Flight Center, NSSTC, XD-12, 320 Sparkman Drive, Huntsville, AL 35805, USA

14 Department of Astrophysics, Radboud University, PO Box 9010, 6500 GL Nijmegen, The Netherlands 